\title{
Anti-EGFR function of EFEMP1 in glioma cells and patient prognosis
}

\author{
Yuanjie Hu${ }^{1}$, Hengjun Gao ${ }^{2,3}$, Christopher Vo ${ }^{4}$, Chao $\mathrm{Ke}^{4,5}$, Francine Pan ${ }^{4}$, Liping \\ $\mathbf{Y u}^{6}$, Eric Siegel ${ }^{7}$, Kenneth R. Hess ${ }^{8}$, Mark E. Linskey ${ }^{4}$, Yi-Hong Zhou ${ }^{1,4}$ \\ ${ }^{1}$ Department of Biological Chemistry, University of California Irvine, Irvine, CA, USA \\ ${ }^{2}$ Institute of Digestive Disease, Tongji University, Shanghai, China \\ ${ }^{3}$ National Engineering Center for Biochip at Shanghai, Shanghai, China \\ ${ }^{4}$ Neurological Surgery, University of California Irvine, Irvine, CA, USA \\ ${ }^{5}$ State Key Laboratory of Oncology in South China and Collaborative Innovation Center for Cancer Medicine, Sun Yat-sen \\ University Cancer Center, Guangzhou, China \\ ${ }^{6}$ Ziren Research LLC, Irvine, CA, USA \\ ${ }^{7}$ Department of Biostatistics, University of Arkansas for Medical Sciences, Little Rock, AR, USA \\ ${ }^{8}$ Department of Biostatistics, The University of Texas MD Anderson Cancer Center, Houston, TX, USA \\ Correspondence to: Yi-Hong Zhou, email: yihongz@uci.edu
}

Keywords: EFEMP1, EGFR, glioma, prognosis, tumorigenicity

Received: January 30, $2014 \quad$ Accepted: March 24, $2014 \quad$ Published: March 25, 2014

This is an open-access article distributed under the terms of the Creative Commons Attribution License, which permits unrestricted use, distribution, and reproduction in any medium, provided the original author and source are credited.

\section{ABSTRACT:}

EGFR is one of the key oncogenes subjected to targeted therapy for several cancers, as it is known to be amplified and/or mutated in up to $40 \%$ of malignant gliomas. EFEMP1, a fibulin-like extracellular protein, exerts both tumor suppressive and oncogenic effects in various cancers and glioma cell models. Although EFEMP1's anti-cancer activity has most commonly been attributed to its anti-angiogenic effects, we showed for gliomas that EFEMP1's binding to EGFR accounts for its suppression of the intracranial tumorigenicity of glioma cells expressing high levels of EGFR. In gliomas where EFEMP1 expression, and thus the anti-EGFR effect of EFEMP1, was suppressed, heightened levels of EGFR expression were associated with unfavorable patient outcomes in prognostic models. Results from the current study clearly demonstrate the impact that the anti-EGFR function of EFEMP1 has on the expression of EGFR and patient prognosis. A glioma prognostic model also suggests EFEMP1's context-dependent oncogenic function in gliomas expressing low levels of EGFR. Hence the level of EFEMP1 expression may have a predictive value for choosing patients for anti-EGFR therapy.

\section{INTRODUCTION}

EFEMP1 is a member of the fibulin family of secreted glycoproteins containing a series of epidermal growth factor (EGF)-like modules, followed by a carboxyterminal fibulin-type module. Fibulins are hypothesized to function as intramolecular bridges within the extracellular matrix to form supra-molecular structures, and as mediators for cellular processes and tissue remodeling, and hence can be involved in cancer [1-3]. Studies of Efemp1 knock-out mice have demonstrated the role of Efemp1 in maintaining tissue integrity, stimulating the expression of Timp1 and Timp3, and inhibiting the expression and activity of matrix metalloproteinases Mmp2, and Mmp9 $[4,5]$. In humans, EFEMP1 was initially identified as a senescence protein $[6,7]$. Malfunction or deregulated expression of EFEMP1 has been implicated in retinal dystrophy, Werner syndrome, adult height, and cancer (see review in [8].

The anti-cancer properties of EFEMP1 were initially thought to be derived from direct targeting of endothelial cell proliferation to suppress angiogenesis 
in cancer [9]. The tumor suppressive role of EFEMP1 has been confirmed in cancers from several organs, including brain, breast, colon, lung, liver, nasopharynx, and prostate [10-16]. Anti-cancer correlations with improved patient prognosis have been based on findings of hypermethylation of the EFEMP1 gene [10-16], downregulated EFEMP1 expression in cancer specimens and derived cancer cell line [10-12, 15-16], and positive correlations of EFEMP1 expression with suppressed lymph-node metastasis $[14,16]$ and overall survival [11-17]. Anti-cancer effects have also been confirmed in vitro through characterization of EFEMP1 function in cell lines derived from human lung cancers [10, 18], nasopharyngeal carcinomas [14], and malignant gliomas [17]. In addition to observation of angiostatic effects, additional mechanisms underlying EFEMP1's tumor suppression function that have since come to light include attenuation of EGFR/AKT-mediated growth signaling activities $[14,17]$ and reduction of MMP-induced cancer cell invasion [18].

A potential oncogenic role of EFEMP1 was identified in cervical carcinoma [19] and pleural mesothelioma [20], and has been found in a human pancreatic carcinoma-derived cell line [21], a chemically induced rat glioma cell line, and in human gliomaderived stem-like glioma cells $[22,23]$. The above reported findings showed dual functions of EFEMP1 in cancer as being both tumor suppressive and tumor promoting. Within specific cancer cell contexts, EFEMP1 may promote [24] or suppress [14, 17] cell growth, by enhancing or reducing AKT phosphorylation, respectively.

Consistent with its role as an extracellular matrix protein, EFEMP1's function in cancer was demonstrated to be an effect in the extracellular compartment, by using a purified human recombinant EFEMP1 protein $[9,17,24]$. These observations suggested that EFEMP1 might have potential benefits in cancer therapy, because its effects appear not to be dependent upon intracellular incorporation, and because the kinetics of extracellular incorporation of a therapeutic agent are far more consistent and predictable than those of intracellular incorporation.

Given the potentially conflicting roles of EFEMP1 for cancer biology in general, and the importance of the EGFR signaling pathway in glioma biology, we studied its suppression of EGFR signaling pathways and tumorigenicity in different glioma cells with or without expression of EGFR, and the prognostic values in different sets of glioma patients, dichotomized based on the expression level of EGFR. Overall data from glioma experimental models and the patient prognosis values consistently demonstrates that EFEMP1 is tumorsuppressive in gliomas driven by activation of EGFR signaling pathways.

\section{RESULTS}

\section{EFEMP1 maintains low levels of EGFR signaling activity and tumorigenesis}

In a prior study, we showed that human recombinant (hr) EFEMP1 protein reduced the EGFR level and AKT phosphorylation (pAKT) in 48-hr treated glioma cell lines [17]. Here show a dose- and time-dependent reduction of EGFR by hrEFEMP1 in one of the studied glioma cell lines, U251 (Figure 1A). Due to batch-to-batch variations of hrEFEMP1 production, which hindered the study of signaling pathways affected by EFEMP1, we made a lentiviral vector that expresses ectopic EFEMP1 (with or without an N-terminal FLAG tag), and infected multiple human high-grade-glioma-derived cell lines (U251, U87, LN229), and a glioblastoma-derived primary culture (G43-SA). Immunoblots showed a high level of EGFR in these glioma cells. The lentiviral construct co-expresses red fluorescent protein (RFP) and EFEMP1 after exposure to doxycycline (Dox), shown by immunofluorescence with antibodies against FLAG and/or EFEMP1 (Figure 2). An increase of overall level of EFEMP1 mRNA following 2472 hours induction with Dox was also verified by real-time qRT-PCR.

As shown in Figure 1B, a 3-day induction of ectopic EFEMP1 (without FLAG tag) caused marked reductions of EGFR in LN229, U87, and G43-SA, and a minor reduction of EGFR in U251, compared to the controls, which were either empty-vector-infected cells or cells without Dox induction (verified by fluorescent microscope to lack RFP). A marked reduction of membrane EGFR in U251 was found, and is shown in the following report, which is consistent with reduction of EGFR signaling targets pERK and pAKT, as shown in other cell lines.

In contrast to a minor reduction of EGFR by a transient EFEMP1 overexpression in U251 cells in vitro, we found significantly suppressed subcutaneous (s.c.) tumorigenesis from a stable induction of ectopic EFEMP1 in U251 and correspondingly, marked reductions of EGFR, in these s.c. xenografts (Figure 1C). We have previously shown tumor suppression from stable transfection of EFEMP1 that nearly abolished U251's tumorigenicity in both s.c. and intracranial (i.c.) xenograft model systems [17]. Here we showed a similar tumor suppressive effect from Dox-induced overexpression of EFEMP1 in glioma cell line U87 and primary culture G43-SA, based on a comparison with the empty-vector control (Figure 1D). 


\section{EFEMP1 interacts with EGFR, blocking EGF from binding and stimulating EGFR signaling}

Given the modular structure of EFEMP1, which has five EGF-like modules and one EGF-like module with insertion [8], we examined interactions between EGFR and endogenous EFEMP1 in U251 and exogenous EFEMP1 in two stable U251 transfectants, with EFEMP1 carrying FLAG tags at either the $C$ - or $N$-terminus (E-CF and E-NF, respectively) (refer to [17] for detailed descriptions). In whole-cell lysates of both EFEMP1-transfectants, EFEMP1 was specifically coimmunoprecipitated by antibody against EGFR (Figure $3 \mathrm{~A}$ ), and EGFR specifically by antibody against the FLAG tag (Figure 3B). After the FLAG pull-downs from the whole-cell lysates, FLAG antibody detected a much weaker signaling in E-NF, as compared to that of E-CF. This suggests removal of the FLAG tag along with the signaling peptide during or after the export of E-NF to the extracellular compartment. The similar strong band of EGFR after the FLAG pull-down in both E-CF and E-NF suggests binding of EFEMP1 to EGFR during or after export to the extracellular compartment.

We then examined the effect of transient EFEMP1 overexpression on EGF-mediated activation of the EGFR signaling pathway. First, we examined the competition between EFEMP1 and EGF in binding EGFR, by an EGF-uptake assay, using fluorescently labeled EGF. EGFuptake is the activation of the canonical EGFR-signaling pathway, and is associated with EGFR internalization [25]. As shown in Figure 4A, EFEMP1 suppressed EGF internalization in both of the glioma cell lines (U251 and G43-SA) examined. Consistently, EFEMP1 reduced EGFR internalization in the presence of EGF, as shown by immunofluorescence detection of EGFR in low-dose (20 ng/ml) EGF-treated U251 infected with lentiviral

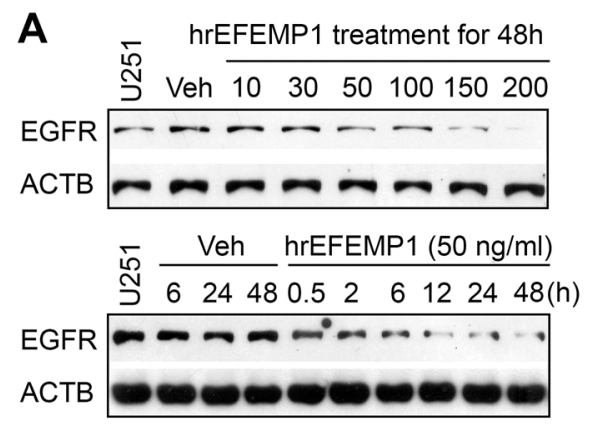

B
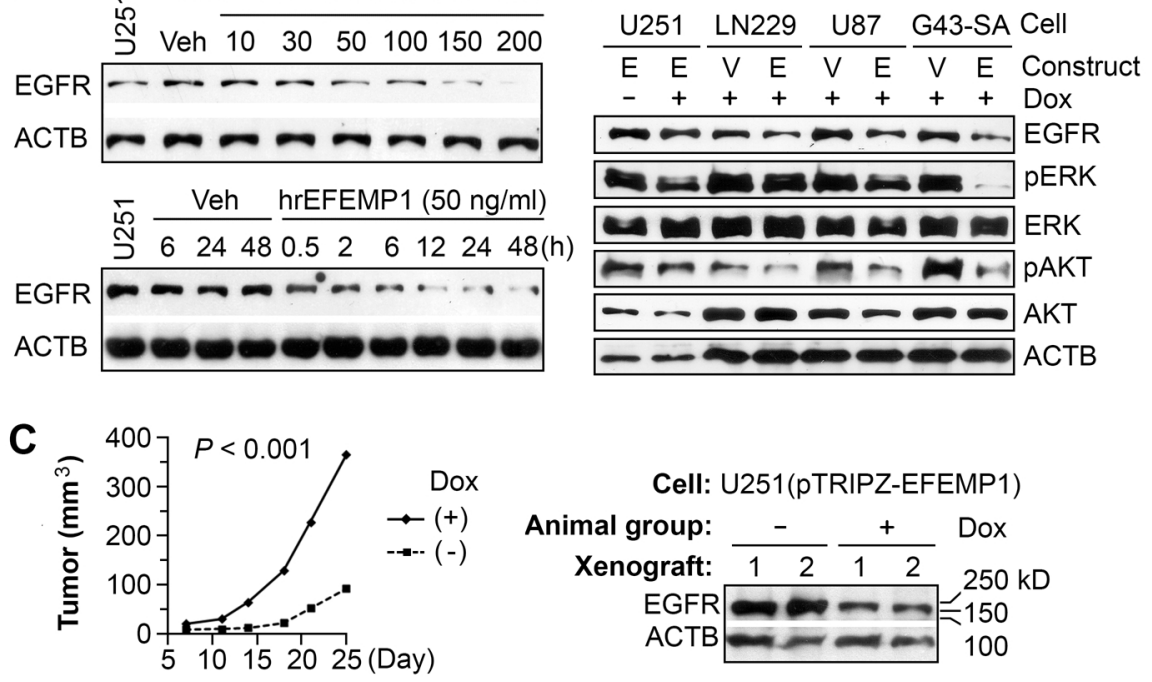

Cell: U251(pTRIPZ-EFEMP1)

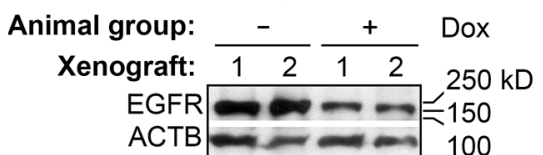

D

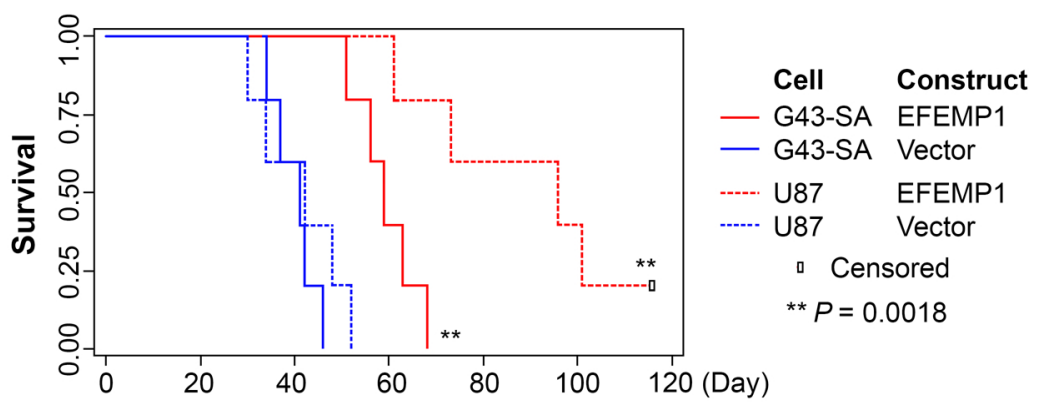

Figure 1: EFEMP1 targets EGFR/EKR/AKT oncogenic pathways to suppress tumorigenicity. (A) Immunoblotting of EGFR in U251 cells treated by human recombinant (hr) EFEMP1 at various concentrations (ng/ml) and for various times (h). (B) Immunoblotting of EGFR and downstream targets in glioma cell lines (U251, LN229, U87) and primary culture (G43-SA) transduced by lentiviral vectors pTRIPZ-Empty (Vector) or pTRIPZ-EFEMP1, with or without a 3-day induction of transgene expression by doxycyclin (Dox, $1 \mathrm{ug} / \mathrm{ml}$ ). (C) Growth curves of subcutaneous (s.c.) xenografts of U251(pTRIPZ-EFEMP1) by measured tumor volumes and western blotting of EGFR in s.c. xenografts with or without providing Dox in water fed to nude mice. (D) Kaplan-Meier survival curves of nude mice with i.c. implantation of EFEMP1- or Vector-transduced U87 and G43-SA (100,000 cells/implantation). 
vector of pTRIPZ-EFEMP1, where ectopic EFEMP1 expression was induced by Dox for 3 days (Figure 4B). Negative controls were infection of empty vector or no Dox treatment.

In the presence of EGF at a low dose $(20 \mathrm{ng} / \mathrm{ml})$, immunoblots of EGFR showed reduction of membranebound EGFR (mEGFR) in no-Dox control cells, but were unchanged in Dox-treated cells (Figure 4C, Lanes $1,2,4$, and 5). A high-dose (100 ng/ml) EGF treatment diminished the effect of EFEMP1 (Figure 4C, Lanes 3 and 6). Overall, the data suggest that EFEMP1, like EGF, binds to EGFR in the extracellular compartment. In contrast to EGF, EFEMP1 inhibits EGFR internalization, but having a long-lasting effect to maintain a low membrane bound EGFR..

We then examined the ability of EFEMP1 to block EGF-mediated activation of EGFR signaling pathways by immunoblotting whole cell lysates of EFEMP1-infected U251 cells. In control cells cultured without Dox, there was canonical EGFR signal activation by EGF, with timedependent reduction of EGFR and a rapid increase and maintenance of high phosphorylation levels for ERK and AKT (solid lines in Figure 4D). In contrast, these striking changes were nearly abolished by expression of ectopic EFEMP1 induced by a 3-day exposure to Dox (dashed lines in Figure 4D).

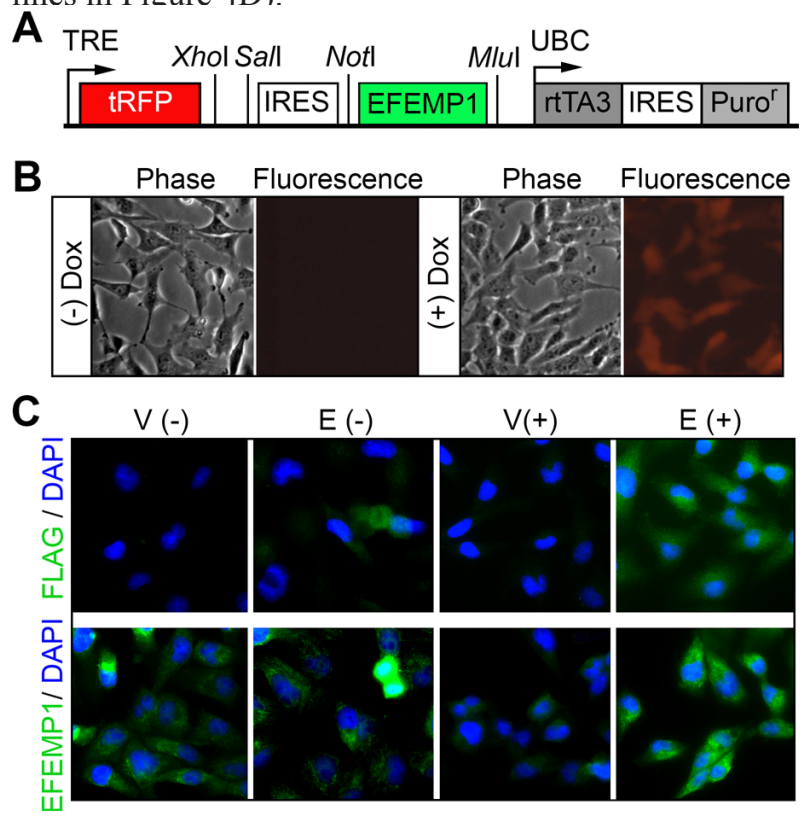

Figure 2: Inducible lentiviral vector overexpressing EFEMP1. (A) Depiction of the pTRIPZ-EFEMP1 construct that co-expresses turboRFP and EFEMP1 (with or without an $N$-terminal FLAG) via the TRE promoter induced by doxycycline (Dox). (upper). (B) Infected glioma cells photographed using an inverted phase-contrast, fluorescence microscope. (C) Immunofluorescence (IF) from antibodies for FLAG and EFEMP1 for ectopic EFEMP1 carrying an $N$-terminal FLAG tag and endogenous/ectopic EFEMP1.

\section{Differential expression of EFEMP1 in gliomas is not correlated with EGFR expression, but does affect patient prognosis}

The oncogenic effect of EGFR overexpression in tumors has been well documented; it is commonly observed in malignant gliomas and, in large part, results from EGFR gene amplifications [26]. However, EGFR expression levels have no prognostic value regarding overall survival of glioma patients [27]. To determine if there is a correlation between protein expression levels of EFEMP1 and EGFR in gliomas, we tested a tissue microarray (TMA) of astrocytic gliomas $(\mathrm{N}=65)$ with antibodies for EGFR and EFEMP1. Comparing to the average of three normal cortex tissues, EGFR was more than 2 -fold over-expressed in $82 \%$ of tumors and more than 10 -fold overexpressed in $77 \%$ of tumors. In contrast, EFEMP1 was under-expressed (less than half of the average of the normal cortex tissues) in $40 \%$ and overexpressed (more than 2-fold of the normal cortex tissues) in $19 \%$ of all tumors, without any significant correlation with tumor grade (Figure 5). Spearman correlation coefficients showed no significant correlation for EFEMP1 and EGFR $(\log 10(\mathrm{~T} / \mathrm{N})$ intensity), with $\mathrm{R}=$ $0.27[95 \% \mathrm{CI}=0.02-0.48 ; P=0.038]$. The significance level was set at $P<0.01$ in order to adjust for the multiple comparisons without overinflating Type II error.

In a published multivariate prognostic model for gliomas, based on quantitative gene expression data, we have shown that the EGFR expression level lacked prognostic value in an astrocytic glioma set comprised of 100 glioblastoma multiformes (GBMs) and 49 anaplastic astrocytomas (AAs), but had a favorable effect on overall survival (OS) of patients with oligodendroglial tumors (OTs, $n=45)$ [28]. In 166 glioma cDNA samples from that study, (95 GBMs, $36 \mathrm{AAs}$, and $35 \mathrm{OTs}$ ), we quantified EFEMP 1 and obtained the absolute ratio of EFEMP 1 to $A C T B$, using same-standard-based, real-time qRT-PCR. Spearman rank correlation coefficient on gene expression data of EFEMP1 and EGFR ( $\log 10$ transformation after a
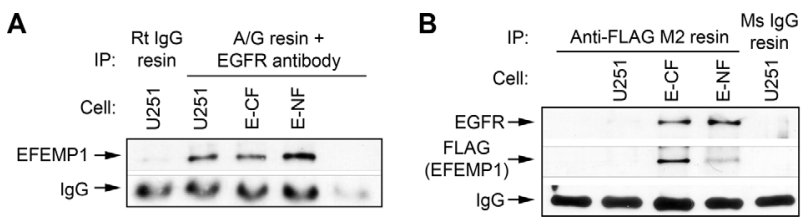

Figure 3: EFEMP1 and EGFR bind in vivo. Coimmunoprecipitation (IP) of EFEMP1 and EGFR in parental or EFEMP1-transfected U251 (E-CF and E-NF refers to FLAG tags at the $C$ - and $N$-terminal ends, respectively), with rabbit anti-EGFR antibody and protein $\mathrm{A} / \mathrm{G}$ beads (A), or mouse anti FLAG M2 affinity gel (B). Rabbit and mouse IgG agarose resin were used as negative controls. 
0.1 offset $(\log 10(\mathrm{x}+0.1))$ was consistent with protein data shown above; with $\mathrm{R}=0.16$ with $[95 \% \mathrm{CI}=0.01-0.31 ; P$ $=0.036]$.

Cox proportional hazards regression analysis was then carried out using both log-scaled ratios of EFEMP1 and EGFR vs ACTB (continuous variables) and dichotomized variables for these 166 gliomas. When EFEMP1 expression was treated as a continuous variable, a negative value $(-0.182)$ of the log hazard ratio (HR), and a $P$ value $=0.097$ in a likelihood ratio test, together suggests a favorable prognostic value for GBM, but lacking prognostic values for AA and OT. We then dichotomized EFEMP1 and EGFR expression variables As shown in Table 1 (left columns), the expression level of $E G F R$ lacked a prognostic value, whereas the expression level of EFEMP 1 correlated significantly with a favorable patient prognosis $(P=0.037)$ in overall gliomas. The favorable prognostic value of EFEMP1 was shown also in $\mathrm{GBM}(\mathrm{HR})=0.7$ and a $95 \%$ confidence interval $=(0.4$ - 1.1), but unfavorable prognostic value of EFEMP1 was implicated in OT, with $\mathrm{HR}=2.4$ and a $95 \%$ confidence interval $=(0.5-11)($ Table 1$)$.

We then analyzed the prognostic value of EGFR in gliomas dichotomized according to high versus low expression level of EFEMP1 and also the prognostic value of EFEMP1 in the same set of gliomas dichotomized according to high versus low expression value of $E G F R$. A log-rank test showed a significant difference among the four groups of gliomas $(P$ value $=0.010)$. In the EFEMP1-low glioma subset $(\mathrm{N}=101), E G F R$ showed a significant, unfavorable, patient prognosis, with $\mathrm{HR}$ $=2.1$ and a $95 \%$ confidence interval $=(1.3-3.4)$. In contrast, in the EFEMPl-high glioma subset $(\mathrm{N}=65)$, $E G F R$ data analysis had no prognostic value (Figure 6AB). In the $E G F R$-low glioma subset $(\mathrm{N}=115), E F E M P 1$ data analysis showed a significant, unfavorable, patient prognosis, with $\mathrm{HR}=1.7$ and a $95 \%$ confidence interval $=(1.1-2.7)$, while in the $E G F R$-high glioma subset $(\mathrm{N}=$ $51)$, the EFEMP1 data lacked any significant prognostic value (Figure 6C-D).

\section{DISCUSSION}

The experimental data described above demonstrated the tumor suppressive function of EFEMP1, which operates by attenuating and blocking EGF-stimulated EGFR signaling activities, and is consistent with the patient outcomes predicted based on the EGFR level in the subset of gliomas expressing a low level of EFEMP1 (Figure 6A). This study extends our prior findings that EFEMP1 exerts an overall tumor suppressive effect in GBM, is an important prognostic variable correlating
A

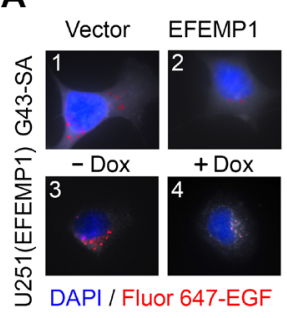

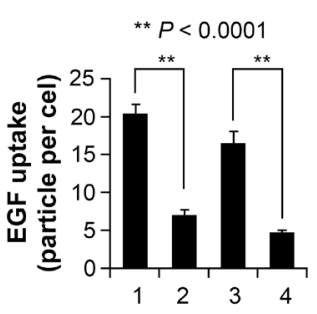
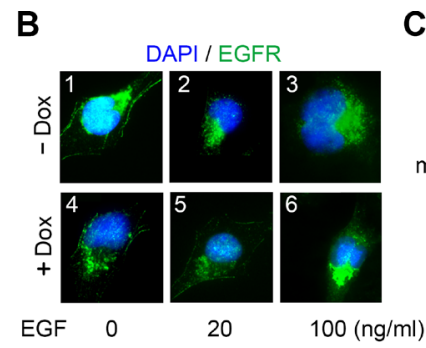

C
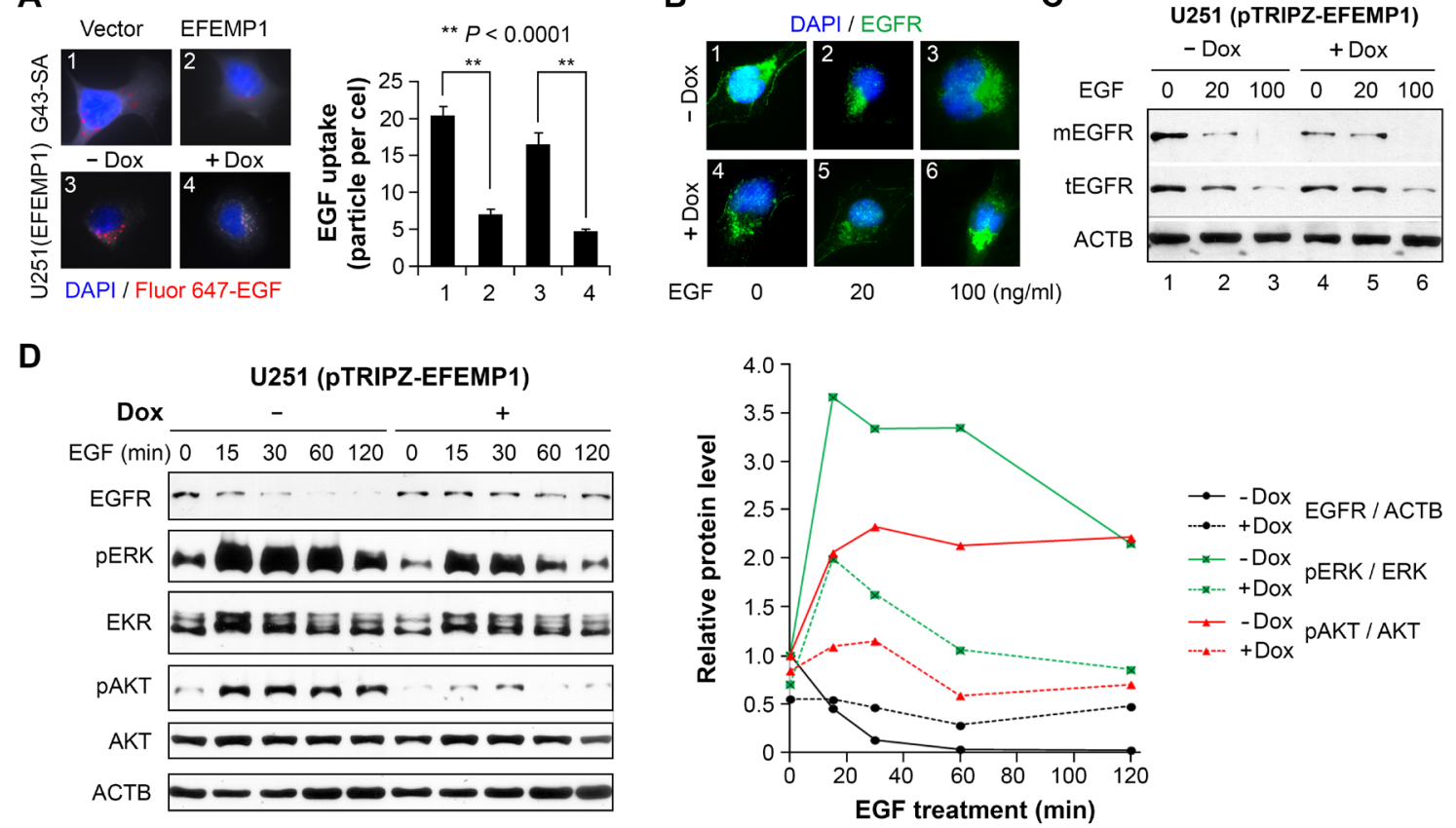

Figure 4: EFEMP1 blocks EGF in activation of EGFR. (A) EGF uptake assay in G43-SA and U251 after a 24-hour treatment with Alexa Fluor 647-EGF. (B) Immunofluorescence of EGFR in U251 after a 24-hour treatment with EGF. (C) Immunoblotting of EGFR in U251 whole cell lysate (tEGFR) and membrane-enriched fraction (mEGFR). (D) Immunoblots (left) and plot of normalized densitometry (right) to show the effect of EFEMP1 (a 3-day induction by Dox) on EGFR activation by a low dose of EGF (20 ng/ml). Cells without Dox at each time point were set to unity. 
with patient survival time, and suppresses tumorigenicity of human glioma cells with high expression level of EGFR in both i.c. and s.c. xenograft models [17]. This effect was reproduced in i.c. xenograft models with another human malignant glioma line, U87, and with a GBM primary culture, G43-SA (Figure 1).

The mechanisms underlying EFEMP1's tumor suppressive function in glioma has been shown to be mediated through EFEMP1's binding to EGFR (Figure 3). Interaction between EFEMP1 and EGFR has also been reported in pancreatic carcinoma cells [24]. However, the consequence of EFEMP1-EGFR binding appears to be organ-specific. In gliomas, our data showed that EFEMP1 binding to EGFR blocked EGF-mediated activation of PI3K and MAPK signaling pathways (Figures 4), while in pancreatic carcinoma cells it was shown to promote these activities [24].

In this study, data from an astrocytic glioma tissue array showed high expression of EFEMP1 in cortical tissues, and de-regulated expression in tumors, with EFEMP1 under-expressed in $40 \%$ of the tissues, over-expressed in $19 \%$ of them and unchanged in the remaining tissues, as compared to the expression level in cortical tissues (Figure 5). These results are different from the finding that Fibulin-3 (EFEMP1) was uniquely up-regulated in malignant gliomas, a conclusion based on microarray data [22]. In the adjacent sections in tissue array analysis, EGFR was demonstrated to have low expression in cortical tissues and to be up-regulated in the majority $(82 \%)$ of tumors; this is consistent with findings of EGFR amplification and overexpression in gliomas that are well documented in the literature and in a recent report from The Cancer Genome Atlas Network (TCGA) [26].

Our prognosis study of glioma patients classified according to EFEMP 1 expression level, demonstrated, for the first time, a significant unfavorable value for $E G F R$ (Figure 6A). However, for gliomas, EGFR's prognostic value can only be properly interpreted in the context of EFEMP1. High EGFR expression is functionally mitigated by high EFEMP1 expression, whereas low EFEMP1 expression identifies the subset of glioma patients for whom anti-EGFR therapies might be the most beneficial. Our finding that EFEMP1 levels in the low-EGFR subset correlate positively with poor patient outcome (Figure 6C). It suggests an oncogenic role of EFEMP1 in gliomas that do not have high EGFR expression, or where tumor growth was not largely driven by EGFRactivated signaling. It has been shown in glioma stemlike cells EFEMP1 activates/depends NOTCH signaling to promote cell invasiveness [23, 29]. By combining

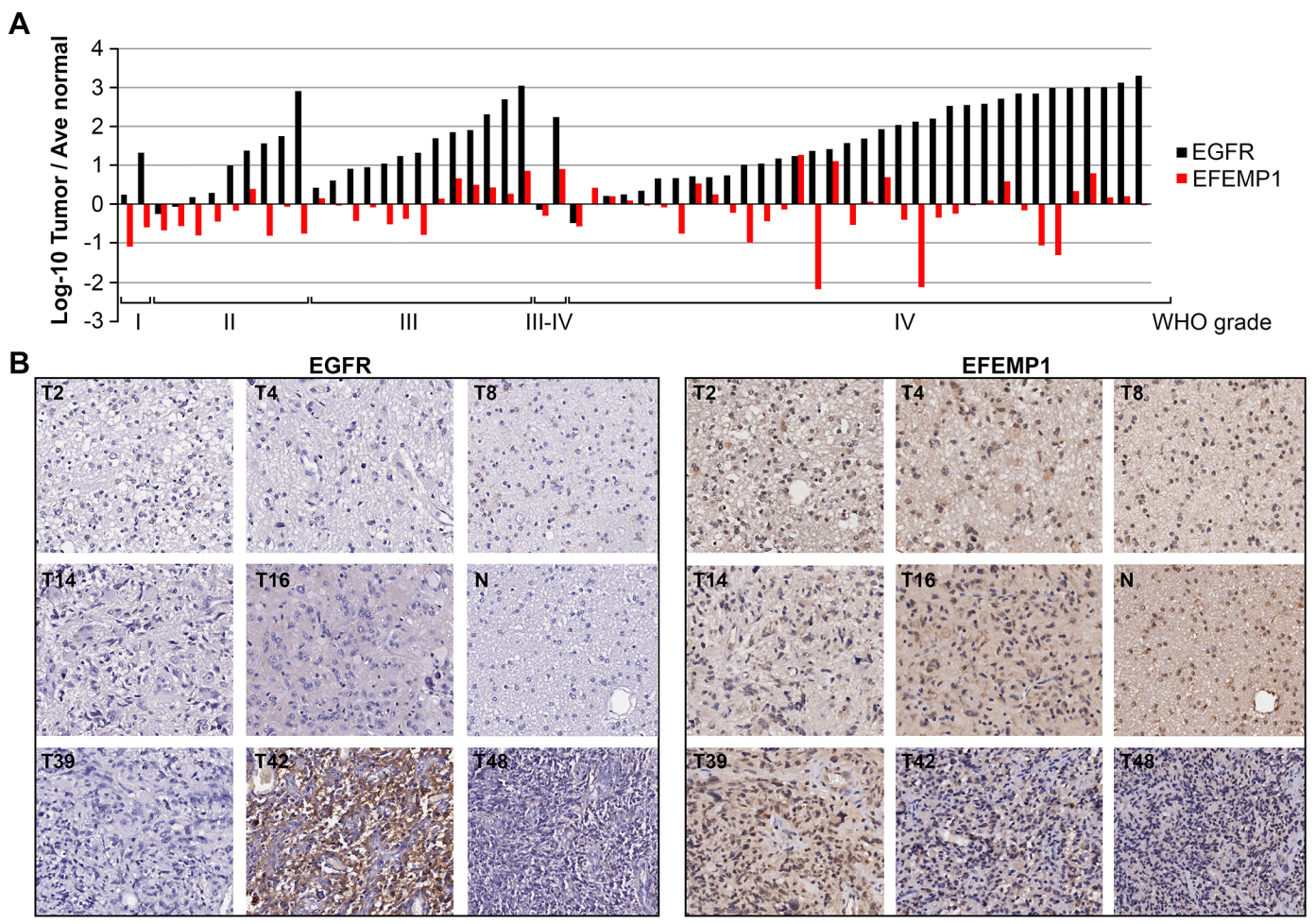

Figure 5: Astrocytic glioma tissue arrays for EGFR and EFEMP1. (A) Relative immunohistochemical intensity of EFEMP1 (red) and EGFR (Black) in 62 gliomas of various grades, according to the World Health Organization (WHO) grading system, normalized to averages of three normal cortical tissues. (B) Representative images of consecutive tissue sections from grade I pilocytic astrocytoma (T2), grade II astrocytoma (T4) and diffuse astrocytoma (T8), grade III anaplastic astrocytoma (T14, T16), normal cortex (N), and grade IV glioblastoma multiforme (T39, T42, T48). 
Table 1: Prognostic effect of EGFR and EFEMP1 in malignant gliomas based dichotomized subtype

\begin{tabular}{|c|c|c|c|c|c|c|}
\hline \multirow{2}{*}{\multicolumn{2}{|c|}{$\begin{array}{l}\text { EGFR/ACTB (cutoff 0.05) } \\
\text { All gliomas a }\end{array}$}} & \multicolumn{5}{|c|}{ EFEMP1/ACTB (cutoff 0.06) } \\
\hline & & All gliomas & & \begin{tabular}{|l} 
GBM \\
(95 pts, 88 died)
\end{tabular} & $\begin{array}{l}\text { AA } \\
\text { (36 pts, } 23 \text { died) }\end{array}$ & $\begin{array}{l}\text { OT } \\
\text { (35 pts, } 18 \text { died })\end{array}$ \\
\hline $1.0(0.7,1.4)$ & $\mathrm{P}=0.85$ & $0.6(0.4,1.0)$ & $\mathrm{P}=0.037$ & $0.7(0.4,1.1)$ & $1.0(0.4,2.3)$ & $2.4(0.5,11)$ \\
\hline
\end{tabular}

${ }^{a}$ Hazard ratio (HR) adjusted by histology, age and recurrent status. The variation is shown with the $95 \%$ confidence interval and $\mathrm{P}$ value from a likelihood ratio test.

findings of poor patient prognosis for gliomas with heightened EGFR levels but low EFEMPI expression, and by clarifying the EGFR-dependent nature of tumor suppression by EFEMP1, our data have provided a rational for low expression of EFEMP 1 being a predictive factor for selecting patients who would benefit from antiEGFR therapy. Anti-EGFR drug is currently being tested in clinical trials using cancer patients [30].

A EFEMP1 low (26 AAs, 46 GBMs, 29 OTs)

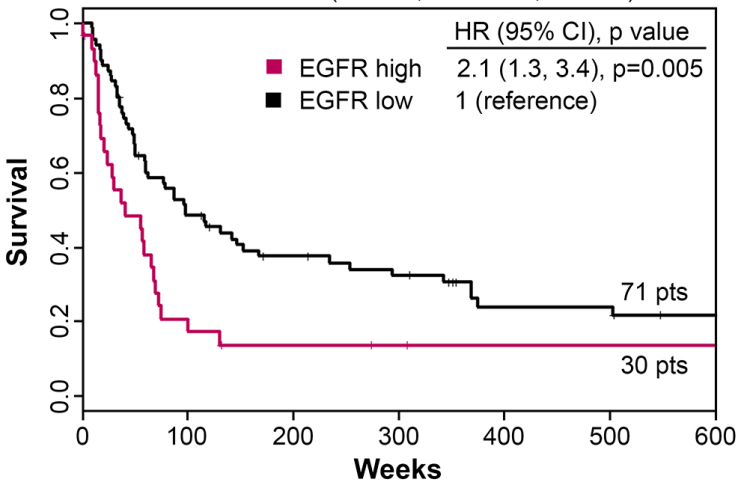

C

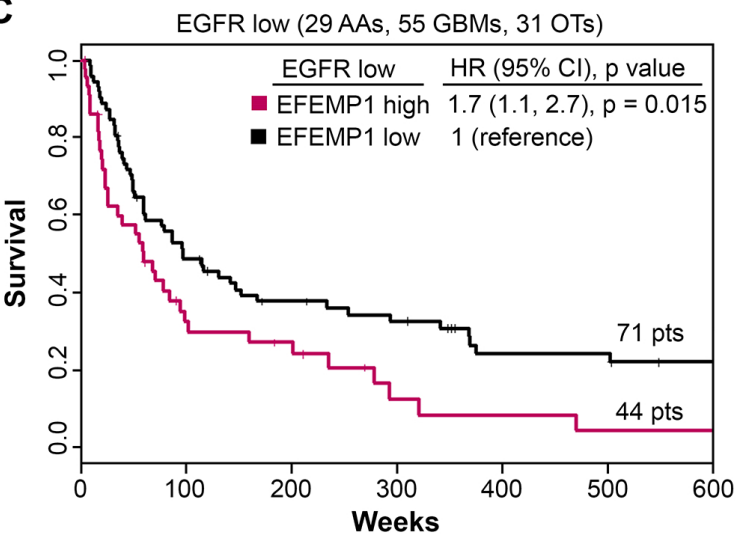

\section{MATERIALS AND METHODS}

\section{Ethics Statement}

Frozen and fresh glioma specimens were provided by the Tissue Banks of University of California, Irvine and Winthrop P. Rockefeller Cancer Institute Bio Repository at UAMS, with Institutional Review Board approval.
B

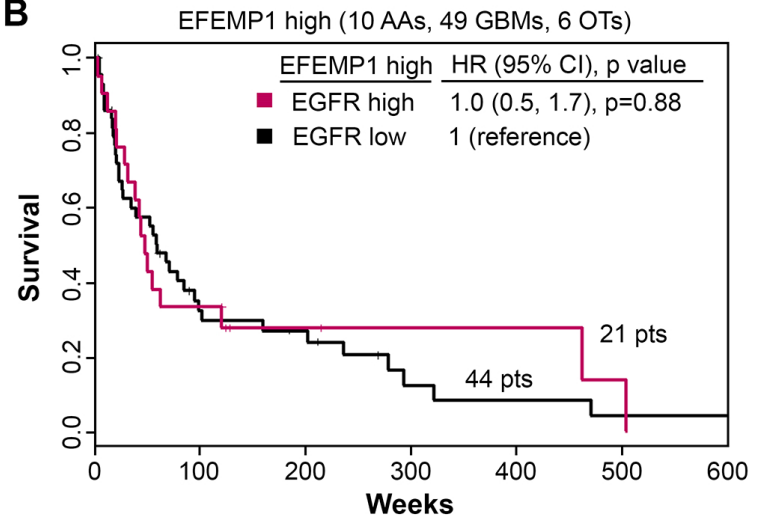

D

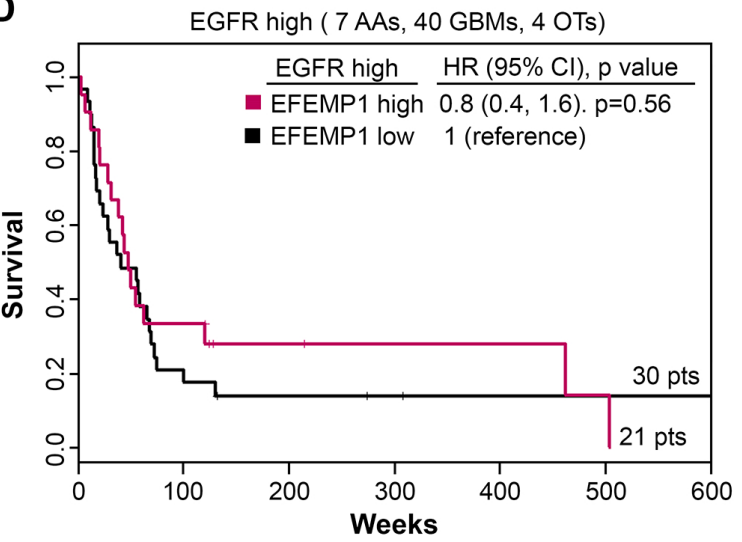

Figure 6: Interdependent prognostic value of $\boldsymbol{E F E M P 1}$ and $\boldsymbol{E} \boldsymbol{G F R}$ expression levels for gliomas. (A-B) Kaplan-Meier survival curves for two groups of glioma patients dichotomized based on EFEMP1 (cut point 0.06 was for EFEMP1/ACTB). Red denotes $E G F R$-high tumors. Black denotes $E G F R$-low tumors. (C-D) Kaplan-Meier survival curves for two groups of glioma patients dichotomized based on EGFR (cut point 0.05 was for $E G F R / A C T B$ ). Red denotes EFEMP1-high tumors. Black denotes EFEMP1-low tumors. 
Animal work on subcutaneous (s.c.) and intracranial (i.c.) xenografts and analysis

The animal work was approved by the Animal Care and Use Committee (IACUC) of the University of California, Irvine. For studies using intracranial (i.c.) xenografts, glioma cells $\left(1 \times 10^{5} / 3 \mu\right.$ l DMEM/F12) were injected into the frontal lobe of 4-6 week old, female, nude mice (strain NCrNu-M, Taconic, Hudson, NY), following IACUC approved surgical procedures. After i.c. implantation, mice were observed daily and periodically weighed for moribund signs (hunchback posture, marked weight loss and gait impairment). Mice were euthanized when they developed brain-damage symptoms (ataxia, hemiparesia, etc) and/or $20 \%$ body weight loss, and the following day was recorded as the survival date for survival analysis.

For studies using subcutaneous (s.c.) xenografts, cells $\left(1 \quad \mathrm{x} \quad 10^{6}\right.$ cells / 50 H1 DMEM/F12) were subcutaneously injected into nude mice, anterior to their right and left thighs, on both sides. Tumor measurements were taken every 3-4 days after implantation, and tumor volume was calculated using the formula $\mathrm{V}=$ $\left(\mathrm{L}^{*} \mathrm{~W}^{2}\right) / 2$ (L, length; $\mathrm{W}$, width). Mice were euthanized at a predetermined time of the experiment or when tumor volume exceeded $1.5 \mathrm{~cm}^{3}$.

Induction of EFEMP1 expression was achieved by providing mice with water containing $1 \mathrm{mg} / \mathrm{mL}$ Dox throughout the experiment. Overall survival of mice bearing intracranial glioma xenografts was estimated using Kaplan-Meier survival curves, and the $P$ values were from Log-Rank statistics on pair-wise comparisons of the two groups using Cox Regression. SAS versions 9.2 and 9.3 (The SAS Institute, Cary, NC) were used for all analyses.

\section{Prognosis analysis of EGFR and EFEMP1 expressions in gliomas}

The cDNA samples of gliomas and quantification of EGFR and EFEMPI have been described in our prior studies [17, 27]. Briefly, we used AqRT-PCR technology [31], with a single standard containing marker and reference genes and gene-specific PCR primers for EFEMP1 and ACTB (Ziren Research LLC, Irvine), to obtain their absolute expression ratios in cDNA samples. In this study, the gene expression data were from 167 glioma cDNA samples. One patient was missing followup data, so analyses were based on 166 patients. The completeness of overall survival (OS) data was shown in our previous study [8]; for glioblastoma multiforme (GBM, WHO grade IV, 92\% deaths, not including those for whom contact had been lost for over 5 years), almost mature for anaplastic astrocytoma (AA, WHI grade III, with $67 \%$ deaths), and half mature for oligodendroglial tumors (OT, 49\% deaths).

Cox proportional hazards regression analysis was done on dichotomized gene expression variables. The cut-offs were chosen so that the group that was high for both markers had at least 20 patients. This was done by inspecting the joint distributions of the two markers. Hazard ratio (HR) values and $95 \%$ confidence intervals were shown for gene expression variables to overall survival of patients.

\section{Glioma tissue microarray (TMA)}

Astrocytic glioma TMAs were fabricated by the Shanghai Outdo Biotech Co., Ltd. The arrays contained 65 individual tissues including pilocytic astrocytoma, astrocytoma, diffuse astrocytoma, anaplastic astrocytoma, normal cortex, and glioblastoma multiforme. IHC was performed on arrayed tissue samples using antibodies of EGFR (Maixin-Bio, MAB0196) and EFEMP1 (Abgent, AP9095a), following standard IHC procedures. Briefly, after deparaffinization and rehydration using a Leica autostainer XL ST5010 system, the TMA slides were pretreated with $10 \mathrm{mM}$ sodium citrate buffer $(\mathrm{pH} \mathrm{6.0)}$ ) for 5-10 minutes in a microwave for antigen retrieval. The endogenous peroxidase was quenched by adding the hydrogen peroxide $\left(3 \% \mathrm{H}_{2} \mathrm{O}_{2}\right.$ in $70 \%$ methanol) at room temperature for 15 minutes. After washing, the slides were blocked for $30 \mathrm{~min}$. The blocking buffer was removed and the slides were then incubated 1 hour with primary antibody at 1:100 dilution at room temperature. Slides were washed with PBS solution and further incubated with DAKO Envision+/HRP for $30 \mathrm{~min}$ at room temperature. Detection was done by 3, 3'-diaminobenzidene (DAB kit, DAKO, Denmark), following the manufacture's protocol. Slides were counterstained with hematoxylin before microscopic analysis.

The expression levels of EGFR and EFEMP1 in TMA were measured by analyzing the staining signal intensity using an Aperio image scope v11 (Aperio, USA). Briefly, the built-in positive pixel count $\mathrm{v} 9$ algorithm was used to measure the densitometry of the digital image (X 400 ), and the counted positive pixels were converted to three intensity bins. The relative log ratio was taken from comparison of individual tissues to the mean of three normal cortical tissues.

\section{Glioma primary cultures and cell lines}

The human glioma cell lines (LN229, U251, and U87) were obtained from Dr. Alfred Yung, M.D. Anderson Cancer Center, University of Texas. GBM-derived primary culture line G43-SA were described in [32], with cell line 
authentication information provided there. LN229, U251, U87, and G43-SA were cultured in Cell Culture/Petri dishes in DMEM/F12 supplemented with $5-10 \%$ fetal bovine serum. All cells were cultured in $37^{\circ} \mathrm{C}$ humidified $\mathrm{CO} 2(5 \%)$ incubators. Apart from LN229, all the other three glioma lines were cultured in serum-free medium for 2 days prior to analyses for proteins in the EGFR signaling pathway, where LN229 remained in culture medium containing serum.

\section{EFEMP1 lentiviral vector construction and lenti virus production}

The protein-coding sequence (CDS) of EFEMP1 cDNA was PCR-amplified by a 5' primer carrying a Kozak site with or without a FLAG tag at the $N$-terminal of CDS, and 3' primer carrying a $X b a$ I restriction enzyme site, cloned into a TA cloning vector. EFEMP1/pCR2.1 clones were selected for carrying a Not I restriction enzyme site upstream of the insertion. After sequencing verification, an error-free EFEMP1-TA clone was subcloned into a modified $\mathrm{pBS}-\mathrm{KS}(+)$ vector with IRES (from pIRES, Invitrogen) inserted upstream of EFEMP1. Then IRESEFEMP1 was subcloned into pTRIPZ-Empty (Open Biosystems) via $X h o \mathrm{I}$ and $M l u \mathrm{I}$. Generation of infectious lentivirus was done by co-infecting HEK-293T with pTRIPZ-Empty (Vector) or pTRIPZ-EFEMP1, along with packaging plasmid psPAX2 and envelope plasmid pCMVVSVG, as described previously [17]. Two days after cotransfection, culture medium containing lentivirus was filtered $(0.45 \mu \mathrm{m})$ and applied to glioma cell cultures. The infected glioma cells were selected for 1-2 weeks in culture medium containing $1.25 \mu \mathrm{g} / \mathrm{ml}$ puromycin prior to analysis.

\section{Co-immunoprecipitation (Co-IP)}

Exponentially growing U251 parental and stable transfectants with FLAG-tagged EFEMP1 were harvested by scraping and sonicated in radioimmunoprecipitation assay buffer (RIPA) containing $1 \mathrm{X}$ cocktail of protease inhibitor (Roche), then centrifuged at $14,000 \mathrm{rpm}$ for $10 \mathrm{~min}$ at $4^{\circ} \mathrm{C}$, and applied to Co-IP using unconjugated EGFR antibody with Protein A/G Plus agarose bead (Santa Cruz Biotechnology, Santa Cruz, CA) or anti-FLAG M2 affinity gel (Sigma-Aldrich, St. Louis, MO), following the manufacturer's protocols. Cell lysate $(\sim 500 \mu \mathrm{g}$ protein in $260 \mu \mathrm{l}$ ) was used for each pull-down, with $5 \mu \mathrm{g}$ of EGFR ( antibody vs protein lysate $=1: 100$ ) antibody and $40 \mu \mathrm{l}$ of $\mathrm{A} / \mathrm{G}$ resin used for EGFR-pull downs, or 40 $\mu 1$ of anti-FLAG M2 affinity gel for FLAG-pull downs. Negative controls for both pull down assays were rabbit or mouse IgG agarose beads (Cell Signaling) added to the cell lysate of $\mathrm{U} 251$, and A/G or anti-FLAG M2 resins added to RIPA buffer. After binding ( $4^{\circ} \mathrm{C}$ overnight), the resins was centrifuged at $6000 \mathrm{rpm}$ for $30 \mathrm{sec}$ at $4^{\circ} \mathrm{C}$. Supernatant fractions were carefully removed. The resin/ bead was washed three times with the cold RIPA or TBS buffer (50 mM Tris-Cl, $150 \mathrm{mM} \mathrm{NaCl}, \mathrm{pH} 7.4$ ) to remove on-specifically bound proteins then eluted in $2 \mathrm{X}$ SDS sample buffer and boiled for $3 \mathrm{~min}$ prior to loading onto SDS-PAGE gels for immunoblotting using antibodies for EFEMP1 (1:500, Aviva Systems), FLAG-M2 (1:1,000, Sigma-Aldrich), and EGFR (1:1000, Cell Signaling)

\section{EGF-uptake assay}

Glioma cells transduced with pTRIPZ-Vector or pTRIPZ-EFEMP1 lentiviral vectors were plated onto ploy-1-lysine $(15 \mathrm{mg} / \mathrm{ml})$-coated chamber slides for $24 \mathrm{~h}$ in DMEM-F12 medium containing 5\% bovine serum. Ectopic EFEMP1 expression was induced by Dox (1ug/ $\mathrm{ml}$ ) for 2 days. Prior to the EGF uptake assay, cells were washed, and incubated in pre-warmed uptake medium (DMEM containing 15mm HEPES, PH7.2, and $1 \% \mathrm{BSA}$ ) for $1 \mathrm{~h}$, then incubated on ice for $30 \mathrm{~min}$ in uptake medium containing $2 \mathrm{ug} / \mathrm{ml}$ of Alexa Fluor 647EGF (Invitrogen), washed with ice-cold PBS and then incubated in pre-warmed uptake medium for $15 \mathrm{~min}$. Cells were placed on ice and incubated in acidic PBS $(\mathrm{pH}=4)$ for $5 \mathrm{~min}$ to remove the unabsorbed fluorescent EGF, then washed twice with ice-cold PBS and fixed in $4 \%$ paraformaldehyde. The intensity of EGF fluorescence was measured by the Image J $1.47 \mathrm{v}$ program (NIH, USA), based on images taken by a fluorescent microscope with a 100X lens. First, the image was imported in TIFF format and then the color channels were separated and converted to gray scale images. The edge of a cell was selected as the region of interest using the brush tool. Ten images for each group were analyzed.

\section{Immunofluorescence and immunoblotting}

U251(pTRIPZ-EFEMP1) cells were grown in chamber slides in culture medium without or with doxycycline $(1 \mathrm{ug} / \mathrm{ml})$ for 2 days, then serumstarved overnight prior to adding EGF to various final concentrations. After a $24 \mathrm{~h}$ EGF treatment, cells were subjected to immunofluorescence detection of EGFR. In brief, cells were rinsed with cold PBS, fixed by 4\% PFA for $10 \mathrm{~min}$ and blocked by $10 \%$ donkey serum for $0.5-1 \mathrm{~h}$ at room temperature, then incubated with EGFR antibody (1:1000 blocking solution) overnight at $4^{\circ} \mathrm{C}$. Alexa $488 \mathrm{~nm}$ donkey anti-rabbit IgG (Invitrogen) was then applied for $1 \mathrm{~h}$ at room temperature, mounted in medium containing DAPI, and then pictures were taken using a fluorescence 
microscope with a $60 \mathrm{X}$ lens.

Similarly treated cells to those described above were subjected to membrane-enrichment by biotin-binding of protein in the cell membrane, followed by streptavidinpull down, using the EZ-Link Sulfo-NHS-Biotinylation Kit (Pierce). Briefly, cells were washed by cold PBS and biotinylated with $0.1 \mathrm{mg} / \mathrm{mL}$ of Sulfo-NHS-LC-Biotin (Pierce) for $30 \mathrm{~min}$ at $4^{\circ} \mathrm{C}$. Then cells were washed with cold PBS containing $0.1 \mathrm{M}$ glycine to quench the unreacted biotin. Cells were lysed in RIPA buffer containing protease inhibitor, and cell debris was removed by centrifugation ( $8000 \mathrm{~g}$ for $10 \mathrm{~min}$ at $4^{\circ} \mathrm{C}$ ). Cleared cell lysates were further incubated with streptavidin-conjugated beads (Pierce) for $45 \mathrm{~min}$ at $4^{\circ} \mathrm{C}$, and the precipitated membrane proteins were then subjected to immunoblotting and compared to the whole cell lysate immunoblotting without biotin-streptavidin precipitation.

Antibodies for EGFR, Akt (AKT), phopho-Akt (Ser473) (pAKT), Erk1/2 (ERK), phopho-Erk1/2 (pERK), and NOTCH1 were from Cell Signaling, and Actin (ACTB) from EMD Bioscience, with dilutions based on the manufacturer's recommendations.

\section{ACKNOWLEDGMENTS}

We would also like to acknowledge Dr. Laura F Hutchins for providing follow-up information for patients from UAMS. We thank Dr. John van der Meer for editing.

\section{Grant Support}

This work was supported, in part, by UC Irvine setup funds and a generous Stern Family gift (YHZ and ML), grants provided by UC Cancer Research Coordinating Committee, UC Irvine Committee on Research, Cancer Center Seed Grant (Award Number P30CA062203 from the National Cancer Institute), Musella Foundation for Brain Tumor Research \& Information, and Voice Against Brain Cancer (YHZ).

\section{Author Contribution}

Conceived and designed the experiments: YZ. Performed the experiments: YH HG CV CK FP LY YZ. Analyzed the data: YH ES KH ML YZ. Wrote the paper: YH YZ.

\section{REFERENCES}

1. Argraves WS, Greene LM, Cooley MA and Gallagher WM. Fibulins: physiological and disease perspectives. EMBO Rep. 2003; 4(12):1127-1131.
2. Gallagher WM, Currid CA and Whelan LC. Fibulins and cancer: friend or foe? Trends Mol Med. 2005; 11(7):336340.

3. Obaya AJ, Rua S, Moncada-Pazos A and Cal S. The dual role of fibulins in tumorigenesis. Cancer Lett. 2012; 325(2):132-138.

4. Rahn DD, Acevedo JF, Roshanravan S, Keller PW, Davis EC, Marmorstein LY and Word RA. Failure of pelvic organ support in mice deficient in fibulin-3. Am J Pathol. 2009; 174(1):206-215.

5. McLaughlin PJ, Bakall B, Choi J, Liu Z, Sasaki T, Davis EC, Marmorstein AD and Marmorstein LY. Lack of fibulin-3 causes early aging and herniation, but not macular degeneration in mice. Hum Mol Genet. 2007; 16(24):30593070 .

6. Lecka-Czernik B, Lumpkin CK, Jr. and Goldstein S. An overexpressed gene transcript in senescent and quiescent human fibroblasts encoding a novel protein in the epidermal growth factor-like repeat family stimulates DNA synthesis. Mol Cell Biol. 1995; 15(1):120-128.

7. Sun BS, Zhu X, Clayton MM, Pan J and Feitelson MA. Identification of a protein isolated from senescent human cells that binds to hepatitis B virus X antigen. Hepatology. 1998; 27(1):228-239.

8. Zhou YH. EFEMP1 (EGF containing fibulin-like extracellular matrix protein 1). Atlas Genet Cytogenet Oncol Haematol 2013; 17.(2):115-120.

9. Albig AR, Neil JR and Schiemann WP. Fibulins 3 and 5 antagonize tumor angiogenesis in vivo. Cancer Res. 2006; 66(5):2621-2629.

10. Yue W, Dacic S, Sun Q, Landreneau R, Guo M, Zhou W, Siegfried JM, Yu J and Zhang L. Frequent inactivation of RAMP2, EFEMP1 and Dutt1 in lung cancer by promoter hypermethylation. Clin Cancer Res. 2007; 13(15 Pt 1):4336-4344.

11. Nomoto S, Kanda M, Okamura Y, Nishikawa Y, Qiyong L, Fujii T, Sugimoto H, Takeda S and Nakao A. Epidermal growth factor-containing fibulin-like extracellular matrix protein 1, EFEMP1, a novel tumor-suppressor gene detected in hepatocellular carcinoma using double combination array analysis. Ann Surg Oncol. 2010; 17(3):923-932.

12. Sadr-Nabavi A, Ramser J, Volkmann J, Naehrig J, Wiesmann F, Betz B, Hellebrand H, Engert S, Seitz S, Kreutzfeld R, Sasaki T, Arnold N, Schmutzler R, Kiechle $\mathrm{M}$, Niederacher D, Harbeck N, et al. Decreased expression of angiogenesis antagonist EFEMP1 in sporadic breast cancer is caused by aberrant promoter methylation and points to an impact of EFEMP1 as molecular biomarker. Int J Cancer. 2009; 124(7):1727-1735.

13. Kim YJ, Yoon HY, Kim SK, Kim YW, Kim EJ, Kim IY and Kim WJ. EFEMP1 as a Novel DNA Methylation Marker for Prostate Cancer: Array-Based DNA Methylation and 
Expression Profiling. Clin Cancer Res. 2011; 17(13):45234530.

14. Hwang CF, Chien CY, Huang SC, Yin YF, Huang CC, Fang FM, Tsai HT, Su LJ and Chen CH. Fibulin-3 is associated with tumour progression and a poor prognosis in nasopharyngeal carcinomas and inhibits cell migration and invasion via suppressed AKT activity. J Pathol. 2010; 222(4):367-379.

15. Tong JD, Jiao NL, Wang YX, Zhang YW and Han F. Downregulation of fibulin-3 gene by promoter methylation in colorectal cancer predicts adverse prognosis. Neoplasma. 2011; 58(5):441-448.

16. Wang R, Zhang YW and Chen LB. Aberrant promoter methylation of FBLN-3 gene and clinicopathological significance in non-small cell lung carcinoma. Lung Cancer. 2010; 69(2):239-244.

17. Hu Y, Pioli PD, Siegel E, Zhang Q, Nelson J, Chaturbedi A, Mathews MS, Ro DI, Alkafeef S, Hsu N, Hamamura M, Yu L, Hess KR, Tromberg BJ, Linskey ME and Zhou YH. EFEMP1 suppresses malignant glioma growth and exerts its action within the tumor extracellular compartment. Mol Cancer. 2011; 10:123.

18. Kim EJ, Lee SY, Woo MK, Choi SI, Kim TR, Kim MJ, Kim KC, Cho EW and Kim IG. Fibulin-3 promoter methylation alters the invasive behavior of non-small cell lung cancer cell lines via MMP-7 and MMP-2 regulation. Int J Oncol. 2012; 40(2):402-408.

19. En-lin S, Sheng-guo C and Hua-qiao W. The expression of EFEMP1 in cervical carcinoma and its relationship with prognosis. Gynecol Oncol. 2010; 117(3):417-422.

20. Pass HI, Levin SM, Harbut MR, Melamed J, Chiriboga L, Donington J, Huflejt M, Carbone M, Chia D, Goodglick L, Goodman GE, Thornquist MD, Liu G, de Perrot M, Tsao MS and Goparaju C. Fibulin-3 as a blood and effusion biomarker for pleural mesothelioma. N Engl J Med. 2012; 367(15):1417-1427.

21. Seeliger H, Camaj P, Ischenko I, Kleespies A, De Toni EN, Thieme SE, Blum H, Assmann G, Jauch KW and Bruns CJ. EFEMP1 expression promotes in vivo tumor growth in human pancreatic adenocarcinoma. Mol Cancer Res. 2009; 7(2):189-198.

22. Hu B, Thirtamara-Rajamani KK, Sim H and Viapiano MS. Fibulin-3 is uniquely upregulated in malignant gliomas and promotes tumor cell motility and invasion. Mol Cancer Res. 2009; 7(11):1756-1770.

23. Hu B, Nandhu MS, Sim H, Agudelo-Garcia PA, Saldivar JC, Dolan CE, Mora ME, Nuovo GJ, Cole SE and Viapiano MS. Fibulin-3 promotes glioma growth and resistance through a novel paracrine regulation of Notch signaling. Cancer Res. 2012.

24. Camaj P, Seeliger H, Ischenko I, Krebs S, Blum H, De Toni EN, Faktorova D, Jauch KW and Bruns CJ. EFEMP1 binds the EGF receptor and activates MAPK and Akt pathways in pancreatic carcinoma cells. Biol Chem. 2009; 390(12):1293-1302.

25. Sorkin A and Duex JE. Quantitative analysis of endocytosis and turnover of epidermal growth factor (EGF) and EGF receptor. Curr Protoc Cell Biol. 2010; Chapter 15:Unit 15 14.

26. Verhaak RG, Hoadley KA, Purdom E, Wang V, Qi Y, Wilkerson MD, Miller CR, Ding L, Golub T, Mesirov JP, Alexe G, Lawrence M, O'Kelly M, Tamayo P, Weir $\mathrm{BA}$, Gabriel S, et al. Integrated genomic analysis identifies clinically relevant subtypes of glioblastoma characterized by abnormalities in PDGFRA, IDH1, EGFR, and NF1. Cancer Cell. 2010; 17(1):98-110.

27. Zhou YH, Hess KR, Raj VR, Yu L, Liu L, Yung AW and Linskey ME. Establishment of prognostic models for astrocytic and oligodendroglial brain tumors with standardized quantification of marker gene expression and clinical variables. Biomark Insights. 2010; 5:153-168.

28. Zhou YH, Tan F, Hess KR and Yung WK. The expression of PAX6, PTEN, vascular endothelial growth factor, and epidermal growth factor receptor in gliomas: relationship to tumor grade and survival. Clin Cancer Res. 2003; 9(9):3369-3375.

29. Hiddingh L, Tannous BA, Teng J, Tops B, Jeuken J, Hulleman E, Boots-Sprenger SH, Vandertop WP, Noske DP, Kaspers GJ, Wesseling P and Wurdinger T. EFEMP1 induces gamma-secretase/Notch-mediated temozolomide resistance in glioblastoma. Oncotarget. 2014; 5(2):363-374.

30. Harandi A, Zaidi AS, Stocker AM and Laber DA. Clinical Efficacy and Toxicity of Anti-EGFR Therapy in Common Cancers. J Oncol. 2009; 2009:567486.

31. Zhou YH, Raj VR, Siegel E and Yu L. Standardization of Gene Expression Quantification by Absolute Real-Time qRT-PCR System Using a Single Standard for Marker and Reference Genes. Biomark Insights. 2010; 5:79-85.

32. $\mathrm{Hu} Y, \mathrm{Ru} N$, Xiao H, Chaturbedi A, Hoa NT, Tian XJ, Zhang H, Ke C, Yan F, Nelson J, Li Z, Gramer R, Yu L, Siegel E, Zhang X, Jia Z, et al. Tumor-specific chromosome mis-segregation controls cancer plasticity by maintaining tumor heterogeneity. PLoS One. 2013; 8(11):e80898. 